\title{
Acute Liver Failure : Management Update
}

\author{
MD. WAHIDUZZAMAN MAZUMDER ${ }^{1}$, FAHMIDA BEGUM ${ }^{2}$, ASM BAZLUL KARIM ${ }^{3}$
}

\begin{abstract}
Acute liver failure (ALF) is a rare but challenging clinical syndrome with multiple causes; about $50 \%$ of pediatric cases a specific etiology cannot be identified. The course of ALF is variable and the mortality rate is high. Liver transplantation is the only therapy of proven benefit, but the rapidity of progression and the variable course of ALF limit its use. Management requires a multidisciplinary approach and is directed at establishing the etiology where possible and monitoring, anticipating, and managing the multisystem complications that occur in children with ALF. Excellent intensive care is critical in management of patients with ALF. Nonspecific therapies are of unproven benefit. Future possible therapeutic approaches include $\mathrm{N}$-acetylcysteine, hypothermia, liver assist devices, and hepatocyte transplantation. Advances in stem cell research may allow provision of cells for bioartificial liver support. ALF presents many challenging opportunities in both clinical and basic research.
\end{abstract}

Keywords: Acute liver failure (ALF), management.

\section{Introduction}

Pediatric acute liver failure (PALF) is a rare but devastating result of hepatic necrosis in previously healthy individuals. Hepatic function rapidly declines within days to weeks and is often complicated by coagulopathy, hepatic encephalopathy (HE), hypoglycaemia, acute renal failure, sepsis, and gastrointestinal bleeding. These complications can lead to death with mortality rates up to $40 \%$ in the United states. In the absence of spontaneous hepatic regeneration, the only therapeutic option that currently holds potential for long term survival is liver transplantation. Acute liver failure (ALF) is the indication for liver transplantation in between $10-20 \%$ of paediatric recipients in major transplant centers. ${ }^{1}$ The overall incidence of paediatric ALF is low, but the rate of devastating complications and mortality is high. In neonates and infants, metabolic disease are the main cause of ALF for which specific medical therapies may in some instances preclude the need for liver transplantation. ${ }^{2}$ The primary causes of ALF in children include viral hepatitis, drug and toxin-induced liver

1. Assistant Professor, Department of Pediatric Gastroenterology \& Nutrition, BSMMU.

2. Assistant Professor, Department of Pediatric Gastroenterology \& Nutrition, BSMMU.

3. Professor \& Chairman, Department of Pediatric Gastroenterology \& Nutrition, BSMMU.

Correspondence: Dr. Md. Wahiduzzaman Mazumder, Assistant Professor, Department of Pediatric Gastro-enterology \& Nutrition, BSMMU.. E-mail: mazumdermw@gmail.com disease, metabolic causes, autoimmune disease and idiopathic causes. About $50 \%$ of cases of pediatric ALF have no identifiable cause. ${ }^{3}$ Exact picture of PALF in Bangladesh is not known. A recent study showed that, aetiology of ALF was identified in $71.4 \%$ cases, Viral hepatitis was found to be the underlying cause in $34.6 \%$,Wilson's disease in $31 \%$, galactosemia in $3 \%$ and $\pm_{1}$-AT deficiency in $3 \%$ cases. After treatment $43 \%$ ALF patients survived, $23 \%$ left hospital with risk bond (DORB), and $34 \%$ patients died. ${ }^{4}$ The purpose of this short review is to focus on the early diagnosis \& prompt referral for available management in home and abroad.

\section{Defination}

Acute liver failure is defined as the development of hepatic encephalopathy within 8 weeks of jaundice in a patient who has no prior history of liver disease. ${ }^{2,3}$ This definition is inadequate for children, because the early stages of encephalopathy are difficult to assess, and encephalopathy may not be apparent until terminal stages of ALF in infants. ${ }^{5}$ In an effort to address the ambiguity associated with the definition of ALF in children, the Pediatric Acute Liver Failure Study Group (PALFSG) which was formed in 1999 came to a consensus regarding the definition of ALF in children. The PALFSG defined ALF as 1) no history of known chronic liver disease, 2) biochemical evidence of liver injury, 3) coagulopathy not corrected by parental 
vitamin K administration 4) INR greater than 1.5 if the patient had encephalopathy or greater than 2.0 if the patient does not have encephalopathy. ${ }^{6}$

\section{Pathogenesis}

The pathologic features of ALF include rapid and massive hepatocyte destrtuction, with failure of the remaining hepatocytes to regenerate. The mechanism of the severe hepatic necrosis is unknown. With severe hepatocellular injury, liver metabolic functions are impaired. Patients have compromised glucose homeostasis, increased lactate production, impaired synthesis of coagulation factors, and reduced capacity to eliminate drugs, toxins, and bilirubin ${ }^{2}$. As a result, patients develop coagulopathy, hypoglycemia, and acidosis, all of which increase the risk of gastrointestinal bleeding, seizures, and myocardial dysfunction. Bacterial and fungal infections often complicate ALF. The leading causes of death in ALF are cerebral edema and sepsis. ${ }^{7,8}$ Respiratory distress requiring mechanical ventilation and/or acute renal failure are also common as are bacterial and fungal infections. ${ }^{9}$

\section{General management principles}

Proper patient management needs to be conducted along multiple parallel paths. ${ }^{10,11}$

- Evaluation for the causes of pediatric acute liver failure,

- Monitoring of the functions of each organ system,

- Identification and treatment of complications,

- Providing medical support to maximize health and survival.

- Establishing an intravenous line to monitor and maintain glucose levels

- Contact and likely transfer to a pediatric intensive care unit (PICU)

- Pediatric intensive care unit (PICU)-consultations should include specialists in pediatric gastroenterology and/or hepatology, pediatric neurology.

Management of PALF requires initial admission to a PICU in most of the cases. This will allow close monitoring of the patient, particularly for changes in mental status. Careful and frequent bed side assessment by an experienced nurse or clinician is essential, and cannot be replaced by indirect monitors such as a cardiorespiratory and oxygen saturation monitor. Care givers must carefully examine the child several times during the day and night to assess-

- Evidence of changing mental status or hepatic encephalopathy,

- Increased respiratory effort,

- Changing heart rate,

- Changes in blood pressure, that might be signs of infection, increasing cerebral edema, or electrolyte imbalance,

- Fluid balance (input and output) should be strictly monitored. ${ }^{10,11}$

\section{Laboratory monitoring}

Laboratory monitoring should include a complete blood count, electrolytes, renal function tests, glucose, calcium, phosphorous, ammonia, PT, INR, total and direct bilirubin, and blood cultures. The frequency of laboratory monitoring should be at least daily, but multiple tests obtained regularly throughout the day may be necessary to monitor the dynamic changes that can occur in PALF. ${ }^{10}$

\section{Fluids}

If the child is in shock, fluid resuscitation and pressor support is needed to stabilize cardiovascular status. However, as a general rule, intravenous and oral fluid intake should be modestly restricted for most patients with PALF. Total daily fluid intake (including medications and blood products) should initially be restricted to between 85 to 95 percent of the maintenance fluid requirement. Patients with PALF are sensitive to fluid volume and can develop pulmonary and peripheral edema if they receive excessive fluid. Serum glucose should be maintained between 90 and $110 \mathrm{mg} / \mathrm{dL}$. A central venous catheter may be required if concentrations of intravenous glucose over 12.5 percent are needed to maintain the serum glucose while restricting fluid volume. Adjustment in fluid rates is based upon the clinical conditions. ${ }^{11}$

\section{Encephalopathy}

Hepatic encephalopathy (HE) is a neuropsychiatric syndrome associated with hepatic dysfunction. ${ }^{12} \mathrm{HE}$ is determined by serial clinical evaluations of behavior, cognition, neurological examination, and, occasionally, electroencephalogram (EEG) to categorize the patient into one of five clinical stages of encephalopathy, ranging from stage 0 (minimal or no evidence of neurological dysfunction) to stage IV (coma). ${ }^{13}$ 
Initial treatment of $\mathrm{HE}$ includes minimizing excess stimulation, head elevation up to 30 degrees, treating suspected sepsis, and, if possible, removing sedative medications that might affect mental status. For patients with progressive $\mathrm{HE}$, medical therapy with lactulose is used empirically although there is only weak evidence to suggest that it is effective. The starting dose of lactulose is 0.4 to $0.5 \mathrm{~g} / \mathrm{kg}$ every two hours by mouth or via nasogastric tube, with the dose adjusted as needed to produce two to three soft stools daily. ${ }^{14}$ Bowel "decontamination" with rifaximin or neomycin can be used as a second-tier treatment, but ototoxicity and nephrotoxicity are potential risks when neomycin is used. ${ }^{15}$ Oral metronidazol is now a days being used for bowel decontamination. Initial restriction of protein intake to no more than $1 \mathrm{~g} / \mathrm{kg} /$ day from stage three of $\mathrm{HE} .{ }^{14}$

Some patients with HE develop a clinically important increase in intracranial pressure, which can have devastating consequences. Direct monitoring of intracranial pressure is the most sensitive and specific procedure to use when compared with less invasive neuroradiographic procedures, such as cranial computed tomography (CT) or transcranial ultrasonography.

Children with ALF may experience generalized or focal seizures, or nonconvulsive (electrographic) seizures (NCS). In most cases, treatment begins with phenytoin, but practices are variable and there is no definitive standard of care. ${ }^{14}$ For seizures which are refractory to phenytoin, therapeutic options include midazolam infusion, phenobarbital, levetiracetam, or topiramate. The selection of drug depends on the patient's mental status, physiologic stability, availability of EEG monitoring to titrate drug infusions, and institutional experience. ${ }^{14}$

\section{Cerebral edema}

Cerebral edema is a life threatening complication of ALF. It may lead to ischemic and hypoxic brain injury, or brainstem herniation and death. ${ }^{15}$ It occurs most commonly in those with advanced hepatic encephalopathy (stage III or IV) and can progress rapidly. Detection of cerebral edema in the early stages is difficult, because noninvasive monitoring with clinical assessment or radiographic studies lacks sensitivity. The most sensitive measure of intracranial pressure (ICP) requires surgical placement of an ICP monitor. ${ }^{16}$

Management of cerebral edema involves meticulous supportive care to maintain the following goals. ${ }^{17}$
- Oxygen saturation above 95 percent

- Total daily fluid between 85 and 90 percent of maintenance

- Maintain a diastolic pressure $>40 \mathrm{mmHg}$ (except in infants)

- Minimize agitation

- Head elevation of $20^{\circ}$ to $30^{\circ}$

- Consideration of empiric broad spectrum antibiotics to minimize the development of bacterial infection. In children with PALF, infection sometimes presents with neurological deterioration, rather than typical symptoms of infection. ${ }^{17}$

Therapies targeted specifically to improve cerebral edema include hypertonic saline to maintain serum sodium between 145 and $150 \mathrm{mEq} / \mathrm{L}$, and mannitol ( 0.5 to $1 \mathrm{gm} / \mathrm{kg} / \mathrm{dose}$ ) to maintain serum osmolarity between 300 and $320 \mathrm{mOsm} / \mathrm{L}$ to create a more favourable osmotic gradient to extract water from the brain. Mannitol is also used as a rescue medication for clinical signs of uncal herniation, which includes pupillary dilatation not responsive to light, bradycardia, and hypertension. Hypothermia has been used in adults with acute liver failure with some success. ${ }^{17}$

\section{Coagulopathy}

The prothrombin time (PT) and international normalization ratio (INR) are used to assess the severity of liver injury in the setting of acute liver failure, because these tests reflect hepatic production of clotting factors, particularly factors V and VII, which have the shortest half-lives. However, the PT and INR are not good markers for the risk of bleeding in patients with ALF. This is because ALF reduces both procoagulant proteins (eg, factor V, VII, X, and fibrinogen) and anticoagulant proteins (eg, antithrombin, protein $C$, and protein S). ${ }^{11,13}$ This balanced reduction in the procoagulant and anticoagulant proteins may account for the relative infrequency of clinically important bleeding in the pediatric acute liver failure patient in the absence of a provocative event such as infection or increased portal hypertension.

\section{Recommendation's for management of coagulopathy- 11,13}

- A single dose of vitamin K should be administered once to initially assess response of the coagulation profile. 
- Daily administration of vitamin $\mathrm{K}$ is unnecessary.

- Efforts to "correct" the PT/INR with plasma or other procoagulation products such as recombinant Factor VII should be avoided.

- Correction of the PT/INR should be limited to patients with active bleeding or in anticipation of an invasive surgical procedure.

- Similarly, we transfuse platelets only if there is evidence of bleeding, or if a surgical procedure is planned in a patient with very low platelet count.

\section{Ascites}

Ascites develops in some but not all patients with acute liver failure. ${ }^{10}$ Precipitating factors include hypoalbuminemia, excessive fluid administration, and infection.

- The primary treatment is moderate fluid restriction.

- Diuretics should be reserved for patients with respiratory compromise or generalized fluid overload. Overly aggressive diuresis may precipitate hepatorenal syndrome. ${ }^{10}$

\section{Bleeding}

Gastrointestinal bleeding is surprisingly infrequent, given the degree of coagulopathy. This is probably because of a balanced reduction in the procoagulant and anticoagulant proteins as described above.

\section{Hypoglycemia}

Hypoglycemia is caused by impaired hepatic gluconeogenesis and depleted glycogen stores. Hypoglycemia is treated with continuous infusion of glucose, which is infused via a central venous catheter to accommodate the hypertonic solution. Glucose infusion rates of 10 to $15 \mathrm{mg} / \mathrm{kg} /$ minute may be required to achieve stable serum glucose levels. ${ }^{18}$

\section{Hypokalemia}

Hypokalemia may be caused by dilution from volume overload, ascites, or renal wasting and correction depends upon aetiology. Judicious use of fluid, salt and if needed intravenous administration of potassium is the treatment of choice for hypokalamia. ${ }^{11}$ Intravenous potassium chloride may be given as per following schedule ${ }^{19}$

$$
\begin{aligned}
& \text { KCL infusion / } 100 \mathrm{ml} \mathrm{IV} \mathrm{fluid-} \\
& 3 \text { meq }(1.5 \mathrm{ml}) \text { if serum } \mathrm{k}^{+}>3 \mathrm{meq} / \mathrm{L} \\
& 4 \text { meq }(2 \mathrm{ml}) \text { if serum } \mathrm{k}^{+} 25-3 \mathrm{meq} / \mathrm{L} \\
& 5 \text { meq }(2.5 \mathrm{ml}) \text { if serum } \mathrm{k}^{+} 2-2.5 \mathrm{meq} / \mathrm{L} \\
& 6 \text { meq }(3 \mathrm{ml}) \text { if serum } \mathrm{k}^{+}<2 \text { meq / } \mathrm{L}
\end{aligned}
$$

\section{Hypophosphatemia}

Serum phosphorus should be monitored frequently, as hypophosphatemia can be profound. While the mechanism is unknown, hypophosphatemia is presumed to result from increased needs due to active liver cell regeneration. Hyperphosphatemia, often associated with renal insufficiency, is considered a poor prognostic sign. ${ }^{18}$

\section{Infections}

Patients with acute liver failure are susceptible to bacterial infection and sepsis because of immune system dysfunction. Evidence of infection may be subtle, such as tachycardia, gastrointestinal bleeding, reduced renal output, or changes in mental status. Fever may or may not be present. Thus, blood cultures should be obtained with any evidence of clinical deterioration, and antibiotics should be initiated empirically. According to to King's College protocol cefuroxime, amoxicillin and fluconazole should be started to cover both gram positive and gram negative organisms. ${ }^{17}$

\section{Nutrition}

Nutrition support should be maintained to avoid a catabolic state. There is little evidence to support the use of enteral formulas designed for hepatic disease (eg, formulas enriched in branched chain amino acids and low in aromatic amino acids). If it is not safe for the child to receive oral or enteral feeding, intravenous alimentation (parenteral nutrition [PN]) should be initiated. 20

\section{Recommendation's for nutritional management- 20}

- Total fluid input including PN, blood products, and medications should generally be limited to between 85 to 95 percent of the maintenance fluid requirement to avoid excessive hydration.

- Protein input should generally be no more than 1 $\mathrm{g} / \mathrm{kg} /$ day, but this may need to be reduced to 0.5 $\mathrm{mg} / \mathrm{kg} / \mathrm{day}$ for patients with elevated serum ammonia levels.

- Trace metals (trace elements) should generally be eliminated or reduced. This is because copper and manganese are metabolized in the liver. Moreover, chromium, molybdenum, and selenium should be eliminated or reduced if renal disease is also present. 


\section{Liver support}

A number of approaches are being developed to perform some functions of the liver in an attempt to delay or avoid the need for liver transplantation. These include artificial hepatic assist devices (eg, the membrane-adsorbent recirculating system (MARS). ${ }^{21}$ To date, none have been established as a valuable treatment for acute hepatic failure. Plasmapheresis or plasma exchange facilitates for removal of suspected toxins in the blood to facilitate a milieu in which the liver might recover or regenerate, but has not been helpful in the management of children or adults with acute liver failure. While coagulation profiles may improve, the procedure has not been shown to improve neurological outcome or ability of the liver to recover spontaneously. An exception is that for patients with ALF due to Wilson disease, plasma exchange can be valuable because it rapidly removes large amounts of copper. A randomized, doubly masked, controlled trial in pediatric acute liver failure (PALF) demonstrated that intravenous $\mathrm{N}$ acetylcysteine (NAC) was not beneficial in children with non-acetaminophen-induced ALF. ${ }^{21}$

\section{Liver transplant}

Liver transplant decisions in the era before liver transplantation (LT) facilities, the natural history of pediatric acute liver failure was for children to either survive or die. LT interrupts the natural course of PALF, and can save the life of a patient with acute liver failure if he or she has a condition that is not amenable to treatment or fails to respond to treatment. However, because the cause of PALF often is not known, and the course of PALF is difficult to predict, it is likely that some patients may receive $L T$ in situations in which spontaneous recovery may have occurred. LT decisions are difficult because of uncertainty regarding the patient's outcome without transplant, the potential morbidity and mortality of the transplant procedure, and the limited number of organs available. Moreover, long-term outcomes following LT for PALF are less favorable as compared with LT for chronic liver diseases such as biliary atresia. This is likely due to multiple factors, including the severity of illness at the time of $L T$, and the possibility that $L T$ was performed in circumstances in which death was inevitable regardless of $L T$. When living donor $L T$ is considered, the decision also includes consideration of potential risks to the donor 20 .

\section{Prognostic factors}

Because of shortage of liver transplantation facilities, a large number of children's of ALF die without undergoing liver tranaplantation. There are certain prognostic factors that can predict those patients who are unlikely to survive without liver transplantation. Increasing grade of encephalopathy, $>7$ days interval between the onset of prodromal symptoms and encephalopathy, blood glucose $<45 \mathrm{mg} / \mathrm{dl}$, serum bilirubin $>10 \mathrm{mg} / \mathrm{dl}$, and $\mathrm{p}^{\mathrm{H}}<7.35$ or $>7.45$ on admission was found to be associated with increased risk of mortality. ${ }^{22}$

\section{Outcomes}

In the pretransplant era and using an adult definition of acute liver failure, spontaneous survival occurred in 28 percent of patients overall, and only 4 percent of those with stage IV coma. ${ }^{23}$ More recently, with improvements in management of critically ill children, coupled with a more lenient definition of pediatric acute liver failure, outcomes have improved. 24,25 However, it should be noted that all current studies of outcomes are affected by decisions about liver transplantation, because LT interrupts the natural course of PALF. Some children who receive LT may have recovered spontaneously, and some die as a consequence of $L T$ rather than of the underlying PALF. Patient outcome depends on a number of factors including the etiology, disease severity, supportive management, and treatment. However, outcomes vary among children with seemingly similar etiology, disease severity, and treatment. Additional factors are likely involved to explain these variations perhaps including the inflammatory milieu, end-organ damage, immune activation, and potential for liver regeneration. ${ }^{26}$

\section{Conclusion}

ALF in children is a challenging medicosurgical condition that requires early diagnosis, a prompt multidisciplinary approach and intensive care management to identify the aetiology and prevent or treat major complications with the hope that the liver function will recover or liver transplantation can be performed. While improvements in supportive medical care and specific medical therapies over the past decade have had a substantial impact on 
survival, these condition continue to carry a high mortality rate, unless emergency liver transplantation can be performed. In our country the obstacle to optimal management of PALF is delayed referral, minimal paediatric intensive care facility, no facility for liver transplantation and above all financial insolvency. So early diagnosis \& prompt referral for available management in home and for liver transplantation in abroad are the existing options in our country.

\section{References:}

1. Lee WM. Acute liver failure in the United States. Semin Liver Dis 2003;23:217-226.

2. Durand P, Debray D, Mandel R, Baujard C, Branchereau S, Gauthier F. Acute liver failure in infancy: a 14-year experience of pediatric liver transplantation centre. J Pediatr 2001;139: 871-6.

3. Colquhoun SD, Lipkin C, Connelly CA. The pathophysiology, diagnosis, and management of acute hepatic encephalopathy. Adv Intern Med 2001;46(suppl 4):155-176

4. Mazumder MW, Karim MB, Rukunuzzaman M, Rahman MA. Aetiology and outcome of acute liver failure in children: Experience at a tertiary care hospital of Bangladesh. Mymensingh Med J 2016 Jul; 25(3):492-494.

5. Rivera-Penera T, Moreno J, Skaff C. Delayed encephalopathy in fulminant hepatic failure in the pediatric population and the role of liver transplantation. J Pediatr Gastroenterol Nutr 1997;24:128-34.

6. Squires RH Jr, Shneider BL, Bucuvalas J. Acute liver failure in children: the first 348 patients in the pediatric acute liver failure study group. $\mathrm{J}$ Pediatr 2006; 148:652-658

7. Higgins PDR, Fontana RJ. Liver transplantation in acute liver failure. Panminerva Med 2002; 52:93-97.

8. Ostapowicz GA, Fontana RJ, Schiodt FV, Larson A, Davern TJ, Han SBH. Results of a prospective study of acute liver failure at 17 tertiary care centers in the United States. Ann Intern Med 2002; 137:945-954.
9. Wade J, Rolando N, Philpott-Howard J, Wendon J. Timing and etiology of bacterial infections in a liver intensive care unit. J Hosp Infect 2003; 53:144-146.

10. Squires $\mathrm{RH}$, Alonso EM. Acute liver failure in children. In: Suchy FJ Sokol RJ, Balistreri WF (Eds) Liver Disease in Children 4th ed Cambridge University Press, New York 2012;1491-93.

11. Squires RH Jr. Acute liver failure in children. Semin Liver Dis 2008; 28:153-66.

12. Debray D, Yousef N, Durand P. New management options for end-stage chronic liver disease and acute liver failure: potential for pediatric patients. Paediatr Drugs 2006; 8:1-13.

13. Wendon J, Lee W. Encephalopathy and cerebral edema in the setting of acute liver failure: pathogenesis and management. Neurocrit Care 2008; 9:97-102.

14. Stravitz RT, KramerAH, Davern T. Intensive care of patients with acute liver failure: recommendations of the U.S. Acute Liver Failure Study Group. Crit Care Med 2007; 35:2498-508.

15. Kamat $P$, Kunde $S$, Vos $M$. Invasive intracranial pressure monitoring is a useful adjunct in the management of severe hepatic encephalopathy associated with pediatric acute liver failure. Pediatr Crit Care Med 2012; 13:e33-8.

16. Shawcross DL, Wendon JA. The neurological manifestations of acute liver failure. Neurochem Int 2012; 60:662-71.

17. Schmidt LE, Dalhoff K. Serum phosphate is an early predictor of outcome in severe acetaminophen induced hepatotoxicity. Hepatology 2002; 36:659-65.

18. Rolando N, Harvey F, Brahm J. Prospective study of bacterial infection in acute liver failure: an analysis of fifty patients. Hepatology 1990; 11:49-53.

19. Arora NK, Mathur, Ahuja A, Oberoi A : Acute Liver Failure. Ind J Pediatr 2003; 70: 73 - 9.

20. Plauth M, Cabre E, Riggio O, Assis-Camilo M, Prilich M, Kondrup J. ESPEN Guidel ines on Enteral Nutrition: Liver disease Clin Nutr 2006;25: 285-94. 
21. Singer AL, Olthoff KM, Kim H, Rand E, Zamir $G$, Shadek A. Role of plasmapheresis in the management of hepatic failure in children. Ann Surg 2001;234:418-24.

22. Kaur S, Kumar P, KumarV, Sarin SK, Kumar A. Etiology and prognostic factors of acute liver failure. INDIAN PEDIATRICS 2013;50:677-9.

23. Squires $\mathrm{RH}$, Dhawan $\mathrm{A}$, Alonso E. Intravenous $\mathrm{N}$-acetylcysteine in pediatric patients with nonacetaminophen acute liver failure: a placebocontrolled clinical trial. Hepatology 2013; 57:1542-9.

24. Squires $\mathrm{RH}, \mathrm{Ng} \mathrm{V}$, Romero R. Evaluation of the pediatric patient for liver transplantation: 2014 practice guideline by the American Association for the Study of Liver Diseases, American Society of Transplantation and the North American Society for Pediatric Gastroenterology, Hepatology and Nutrition. Hepatology 2014; 60:362-98.

25. Psacharopoulos HT, Mowat AP, Davies M. Fulminant hepatic failure in childhood: an analysis of 31 cases. Arch Dis Child 1980; 55:252-5.

26. Sundaram SS, Alonso EM, Narkewicz MR. Characterization and outcomes of young infants with acute liver failure. J Pediatr 2011; 159: 813-15. 